\title{
Loeys-Dietz syndrome associated with a heterozygous mutation in TGFBR2 in a female infant: A case report
}

\author{
CHUN ZHU, MEILING TONG and XIA CHI \\ Department of Child Health Care, Women's Hospital of Nanjing Medical University, \\ Nanjing Maternity and Child Health Care Hospital, Nanjing, Jiangsu 210004, P.R. China
}

Received March 10, 2020; Accepted September 10, 2020

DOI: $110.3892 /$ etm.2020.9444

\begin{abstract}
Loeys-Dietz syndrome (LDS) is an unusual hereditary connective tissue disease with no clear standard clinical diagnosis. The present report describes a case of an infant diagnosed with LDS at the age of 2 months and 23 days. To the best of our knowledge, this case represents the youngest reported case of LDS in China. Whole exome sequencing revealed a heterozygous variation, c.1441(exon 6)G >A [p.E481k(p.Glu481Lys) (NM_001024847)], located at chr3:30715708 in the transforming growth factor $\beta$ receptor 2 gene. The patient underwent systematic rehabilitation and standard high-risk infant follow-up. Currently, the overall development of the patient continues to improve. The patient can walk with assistance, stand up on her feet unaided, exhibits improved optical line of sight and both eyes can move up and down, side to side (left and right) and track moving objects. The parents have been advised that a further echocardiogram should be performed as soon as possible and regular follow-up sessions with a cardiovascular specialist are ongoing. LDS diagnosis was confirmed in the patient based on collective findings of skeletal system changes, ocular hypertelorism, ocular exotropia, micrognathia and high-vaulted palate, in combination with a specific pathogenic gene mutation.
\end{abstract}

\section{Introduction}

Loeys-Dietz syndrome (LDS) is a rare hereditary connective tissue disorder with autosomal dominant inheritance and is characterized by widespread systemic involvement (1). It was reported that $\sim 25 \%$ of patients diagnosed with LDS have an affected parent and $\sim 75 \%$ of probands have LDS due to a de novo pathogenic variant (2). At present, the pathogenesis

Correspondence to: Dr Xia Chi, Department of Child Health Care, Women's Hospital of Nanjing Medical University, Nanjing Maternity and Child Health Care Hospital, 123 Tianfei Alley, Mochou Road, Nanjing, Jiangsu 210004, P.R. China

E-mail: 264601855@qq.com

Key words: Loeys-Dietz syndrome, transforming growth factor $\beta$ receptor 2, heterozygous variation of LDS is not entirely clear, and most researchers believe that it is associated with mutations in genes of the cytokine family TGF- $\beta$ (3). The most typical clinical triad includes hypertelorism, bifid uvula or cleft palate, and aortic aneurysm with tortuosity (1). The syndrome shares a number of clinical characteristics with the well-documented Marfan syndrome (MFS), but compared with MFS, clinical progression of aortic aneurysm or dissection in patients with LDS is more rapid, prognosis of non-surgical treatment is poor, requiring close monitoring (4). Pre-symptom diagnosis has clinical significance in the treatment and prognosis of patients with LDS. LDS has become a focus of researchers worldwide, leading to improved understanding of the disease (2); however, LDS remains insufficiently studied in China. The present report describes a rare case of a female infant diagnosed with LDS and provides a review of relevant literature.

\section{Case report}

In February 2019, a girl of 2 months and 23 days was admitted to Nanjing Maternity and Child Health Care Hospital (Nanjing, China). During a physical health examination of the patient at the age of 42 days, facial ocular hypertelorism (Fig. 1A), ocular exotropia, micrognathia and high-vaulted palate were evident. The fingers and toes of the patient were long and thin (Fig. 1B and C). The whole muscle tone was low and joint relaxation in the distal limbs was observed. Additionally, the patient presented with contracted finger joints, adduction deformity of the thumbs, difficulty in extending the knuckles in both hands and camptodactyly. The heart rhythm was regular with no murmurs. The outpatient doctor suspected MFS, and recommended whole-exome sequencing and high-risk infant follow-up at Nanjing Maternity and Child Health Care Hospital.

The patient was born following a full-term pregnancy by cesarean delivery. The birth weight was recorded as 3,500 g. The parents did not exhibit special facial characteristics or a history of cardiovascular disease. The patient was diagnosed with genu recurvatum 1 day after birth and immobilized with an orthopedic cast and fixed with a sling. At 2.5 months of age, the patient could not hold her head up, her eyes did not track moving objects and her limbs exhibited low muscle tone. The patient was subsequently provided physical, cognition and language rehabilitation training at the hospital. The results of tandem mass spectrometry showed that the metabolism of 

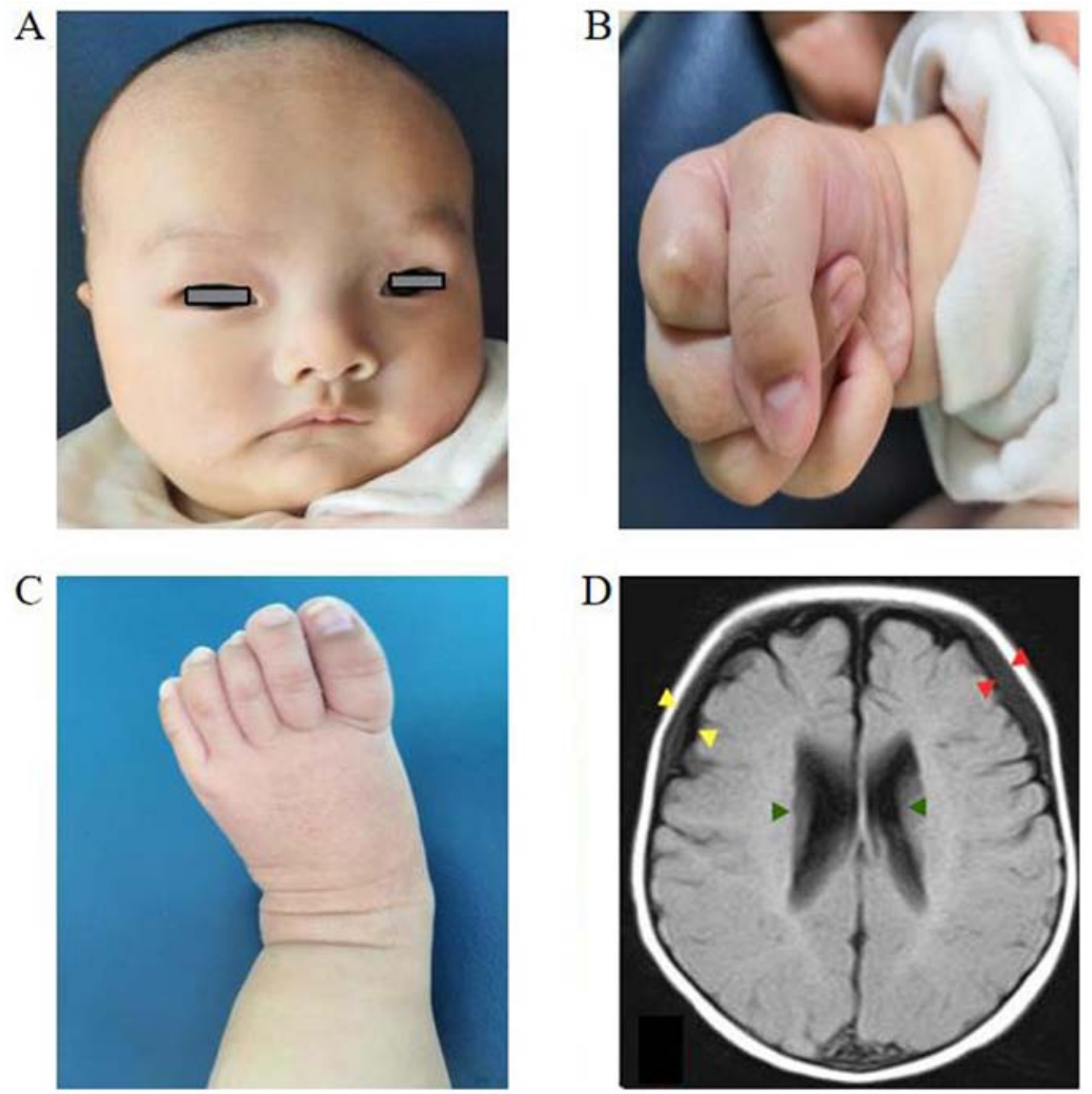

Figure 1. Patient appearance and MRI scan of the head. (A) Ocular hypertelorism. (B) The fingers of the patient were long and thin. (C) The toes of the patient were long and thin. (D) The space between the frontotemporal and parietal brain was widened (yellow arrows), and some subdural effusion in the forehead (red arrows) and dilated bilateral lateral ventricles were observed (green arrows).

Table I. Five types of LDS listed in OMIM.

\begin{tabular}{lccc}
\hline Subtype of LDS & Affected gene & OMIM ID & No. of patients \\
\hline LDS1 & TGFBR1 & 609192 & 135 \\
LDS2 & TGFBR2 & 610168 & 251 \\
LDS3 & SMAD3 & 613795 & 95 \\
LDS4 & TGFB2 & 614816 & 73 \\
LDS5 & TGFB3 & 615582 & 53 \\
\hline
\end{tabular}

LDS, Loeys-Dietz syndrome; OMIM, Online Mendelian Inheritance in Man; TGFB, transforming growth factor $\beta$; TGFBR, transforming growth factor $\beta$ receptor.

organic acids, amino acids and fatty acids was normal (Non derivatization mass spectrometry detection kit; PerkinElmer, Inc.) A brain MRI scan revealed widening of the space between the frontotemporal and parietal brain, some subdural effusion in the forehead and dilated bilateral ventricles (Fig. 1D). No obvious abnormalities were detected by the X-ray examination of both hip joints. Color Doppler echocardiography revealed that the foramen ovale was not fused $(3 \mathrm{~mm})$, and the diameters of the atrioventricular and great vessels were normal. Ophthalmic examination revealed exotropia with no abnormalities in the ocular fundus. Peripheral vein blood was collected from the patient and the parents sent to Chigene
Translational Medical Research Center Co., Ltd. (Beijing, China) for whole exome sequencing. Gene analyses included: i) screening for mutations using high-throughput sequencing technology; ii) data analysis was performed using OMIM (https://omim.org/), Clinvar (https://www.clinicalgenome. org/data-sharing/clinvar/), HGMD (http://www.hgmd. cf.ac.uk/ac/index.php), dbSNP (http://www.bioinfo.org.cn/ relative/dbSNP\%20Home\%20Page.htm) and gnomeAD (Genome Aggregation Database) databases; and iii) verification of suspected pathogenic mutations using Sanger sequencing. The exon capture chip of the xGen Exome Research Panel (version 1.0; Integrated DNA Technologies) 

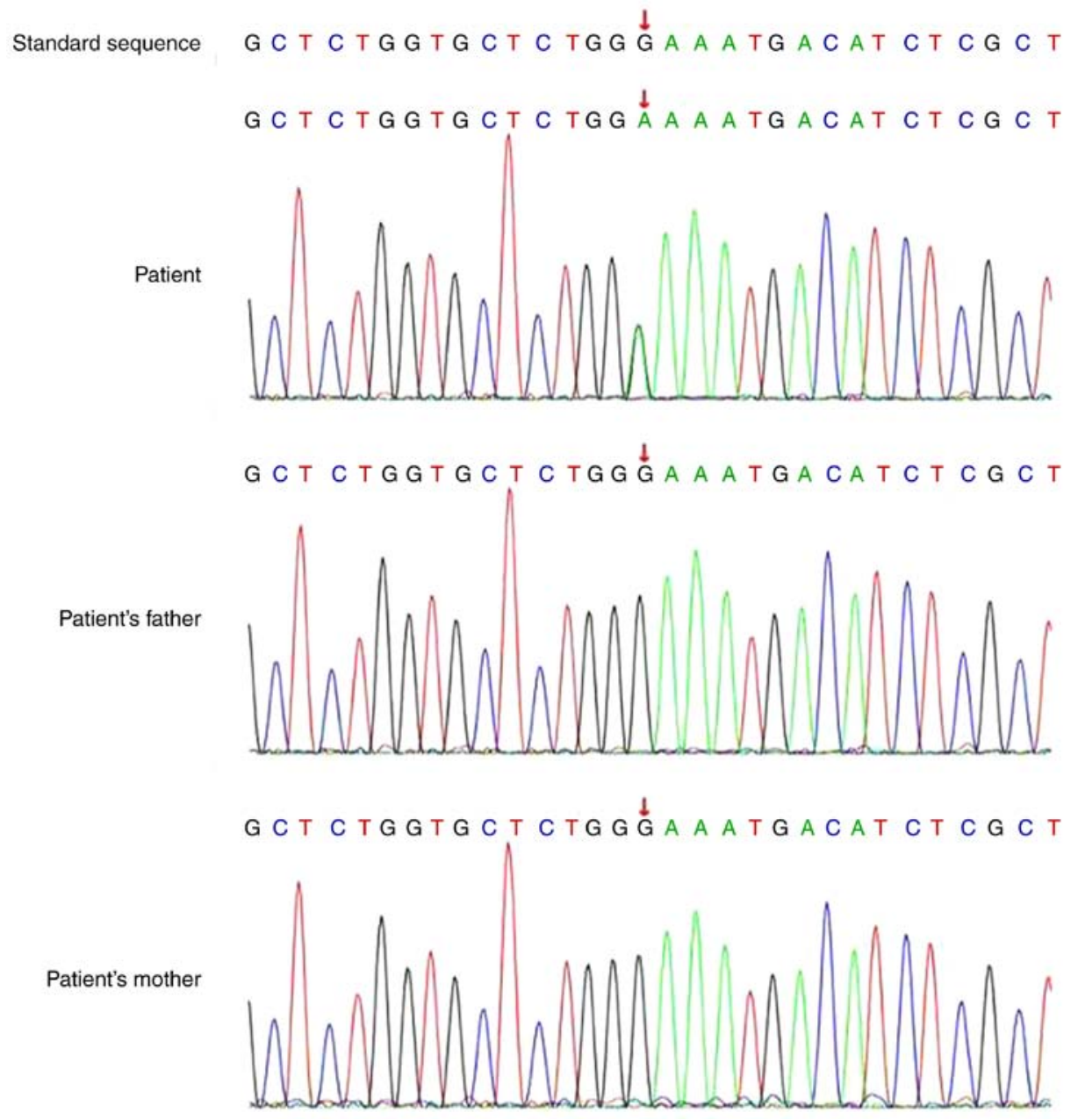

Figure 2. Sequencing of the transforming growth factor $\beta$ receptor 2 gene in the patient and the patient's parents. Arrows indicate TGFBR2:c.1441(exon6)G $>A$.

was applied for whole exon group sequencing, which was completed using the Illumina NovaSeq system 6000 (Illumina, Inc.) series sequencer. Coverage of the target sequence was $\geq 99 \%$. A heterozygous variation, c.1441(exon 6)G $>$ A [p.E481k(p.Glu481Lys)(NM_001024847)], in the transforming growth factor $\beta$ receptor 2 (TGFBR2) gene was identified in the patient. However, neither of the parents carried this mutation (Fig. 2). The diagnosis of LDS was confirmed based on the collective findings. The overall development of the patient continued to improve with systematic rehabilitation. Presently, the patient can walk with assistance, get up on her feet unaided and, in terms of optical line of sight, move her eyes left and right, up and down, and track moving objects. However, responses to different facial expressions are not obvious, eye contact is poor and exotropia still exists. The patient is currently 14 months old and is undergoing regular ophthalmic follow-up. The parents have been advised that a heart B ultrasound should be performed as soon as possible. Regular follow-ups with a cardiovascular specialist have been continued.

\section{Discussion}

LDS was first reported in 2005 and the most typical presentation features alterations in cardiovascular, craniofacial, neurocognitive and skeletal development (5). At present, a total of 619 cases have been documented in the LDS Mutation Database (http://143.169.238.105/LOVD/diseases). While the pathogenesis of LDS remains unclear, mutations in the transforming growth factor $\beta$ receptor 1 (TGFBRI) and TGFBR2 genes identified in 2005 are the first known causative factors (5). Subsequently, other genes in the TGF- $\beta$ signaling pathway, including $S M A D 3$, transforming growth factor $\beta(T G F B) 2$, $S M A D 2$ and $T G F B 3$, have been reported to be associated with LDS (6). Furthermore, LDS is subdivided into five clinical subtypes based on the pathogenic genes involved (Table I).

Clinical presentations of LDS, particularly skeletal features, overlap with those of MFS. Joint relaxation of the distal limbs in the patient was evident and the fingers and toes were long and thin, which could easily be misdiagnosed as MFS (7). Abnormal skeletal features in all types of LDS include pectus deformity, scoliosis and flat feet. Although evidence of skeletal overgrowth predominantly includes arachnodactyly and pectus deformities, height and the proportion of arm to leg length to height are usually within the normal range (8). Furthermore, camptodactyly, talipes equinovarus, contractures of joints and extremity contractures in conjunction with joint hyperextension are common features of LDS (8). Other common manifestations include decreased stability of the cervical spine and anterior displacement of the spine (8). 
LDS is commonly associated with various congenital diseases, including bicuspid aortic valve, atrial septal defect, patent ductus arteriosus and mitral valve prolapse (1). Aortic root dilatation is the most frequent and serious clinical manifestation $(9,10)$. Patients with LDS present with routine involvement of vascular segments distant from the aortic root, which is a more aggressive vascular course than that observed in MFS (4). The mean age of death of individuals with aggressive arterial aneurysms is 26 years (11). Vascular dilatation occurs mainly in the sinus node, which is further prone to aortic dissection or even rupture. A study reported early dissection during a young age in patients with TGFBR1, TGFBR2 or SMAD3 mutations (6).

No specific treatments are currently available for LDS and the primary aim is to prevent vascular accidents and to improve prognosis (8). All patients with LDS require echocardiography at regular intervals (at least once per year or more frequently) to monitor the status of the aortic root, ascending aorta and heart valves (8). At follow-up, the decision to undergo aortic surgery is based on the absolute dimension of the aorta, rate of progression, valve function, severity of non-cardiac features, genotype and family history (8). The skeletal system, and craniofacial and ocular symptoms should be regularly monitored by a multidisciplinary team.

In the current case, the patient presented with clinical manifestations and multiple system abnormalities, including long and thin fingers and toes, contraction of finger joints, deformation of the thumbs and general recurvatum skeletal system changes. Furthermore, the symptoms, including ocular hypertelorism, ocular exotropia, micrognathia and high-vaulted arch, were consistent with the classic symptoms of LDS $(1,5)$.

Due to overlapping clinical phenotypes, no clear standard diagnosis for LDS is available and gene detection is used as an effective diagnostic tool (12). The patient in the present report exhibited a heterozygous c.1441(exon 6)G $>$ A [p.E481k(p. Glu481Lys)(NM_001024847)] mutation in the TGFBR2 gene located at chr3:30715708. This pathogenic abnormality has been documented in the LDS Mutation Database. Based on the clinical manifestations and gene testing results, the patient was diagnosed with LDS. To the best of our knowledge, the patient in the present report is the youngest reported case of LDS diagnosis in China. However, no aorta-related changes were evident in the current case, despite rapidly progressive aortic aneurysmal disease being a distinct feature of LDS (1). Aortic dissection in 3-month-old infants and cerebral hemorrhage in children as young as 3 years has been reported $(13,14)$. The John Hopkins group reported that LDS1 and LDS2 are the more aggressive subtypes and recommend root surgery at a threshold of $4.0 \mathrm{~cm}(15,16)$. Therefore, the present report recommended that parents should ensure continued cardiovascular follow-up for the patient.

Future studies will include a preliminary in vitro experimental study on the mutation site to investigate the impact of the mutation on coding proteins and downstream signaling pathways. It is of great significance to diagnose LDS in the early stage of life, as treatment strategies can be optimized, precise treatments can be designed, and quality of life will be improved. When pediatric patients diagnosed with LDS reach school age, doctors or genetics professionals should provide necessary documentation regarding their diagnosis, physical education restrictions, impact of skeletal and joint features, and the psychological impact of LDS (8). All this information can aid the patient and educational institutions may develop personalized education programs.

In conclusion, LDS is a rare autosomal dominant connective tissue disease involving multiple systems, particularly the cardiovascular system. The progress of LDS is rapid and presents a serious threat to the life of pediatric patients. To further the understanding of the clinical manifestations of LDS, early detection and diagnosis, close monitoring of cardiovascular changes and enhanced follow-up are necessary for suspected cases.

\section{Acknowledgements}

Not applicable.

\section{Funding}

The present study was supported by grants from the National Natural Science Foundation of China (grant no. 81671359), the Subproject of Key Research and Development Program of China (grant no. 2016YFC1000204-6), the Jiangsu Provincial Medical Innovation Team (grant no. CXTDA2017001), The 'Six Talent Peak' High-level Talents Training Project of Jiangsu Province (grant no. WSN-165), the Key Project Supported by Medical Science and Technology Development Foundation Nanjing Department of Health (grant no. zkx18044) and the 333 High Level Talents Training Project of Jiangsu Province.

\section{Availability of data and materials}

All data generated or analyzed during this study are included in this published article.

\section{Authors' contributions}

$\mathrm{CZ}$ collected data, drafted the manuscript and revised the manuscript. MT monitored the data collection and revised the manuscript. XC designed the present study and was responsible for the integrity of the present study. All authors read and approved the final manuscript.

\section{Ethics approval and consent to participate}

The present study was approved by the independent Ethical Committee Review Board of Women's Hospital of Nanjing Medical University, Nanjing Maternity and Child Health Care Hospital (Nanjing, China). Written consent for participation was obtained from the patient's parents.

\section{Patient consent for publication}

Written informed consent for publication of data and images of the patient and their genetic data was retrospectively obtained from the patient's parents.

\section{Competing interests}

The authors declare that they have no competing interests. 


\section{References}

1. Van Laer L, Dietz H and Loeys B: Loeys-Dietz syndrome. Adv Exp Med Biol 802: 95-105, 2014.

2. Loeys BL and Dietz HC: Loeys-Dietz Syndrome. 2008 Feb 28 [Updated 2013 Jul 11]. In: GeneReviews ${ }^{\mathrm{TM}}$ [Internet]. Pagon RA, Adam MP, Bird TD, et al (eds) University of Washington, Seattle, WA, 1993-2013. Available from: https://www.ncbi.nlm.nih.gov/ books/NBK1133/.

3. Ritelli M, Chiarelli N, Dordoni C, Quinzani S, Venturini M, Maroldi R, Calzavara-Pinton P and Colombi M: Further delineation of Loeys-Dietz syndrome type 4 in a family with mild vascular involvement and a TGFB2 splicing mutation. BMC Med Genet 15: 91, 2014.

4. Maleszewski JJ, Miller DV, Lu J, Dietz HC and Halushka MK: Histopathologic findings in ascending aortas from individuals with Loeys-Dietz syndrome (LDS). Am J Surg Pathol 33: 194-201, 2009.

5. Loeys BL, Chen J, Neptune ER, Judge DP, Podowski M, Holm T, Meyers J, Leitch CC, Katsanis N, Sharifi N, et al: A syndrome of altered cardiovascular, craniofacial, neurocognitive and skeletal development caused by mutations in TGFBR 1 or TGFBR 2 . Nat Genet 37: 275-281, 2005.

6. Yang H, Ma Y, Luo M, Zhu G, Zhang Y, Li B, Shu C and Zhou Z: Genetic profiling and cardiovascular phenotypic spectrum in a Chinese cohort of Loeys-Dietz syndrome patients. Orphanet J Rare Dis 15: 6, 2020.

7. Singh KK, Rommel K, Mishra A, Karck M, Haverich A, Schmidtke J and Arslan-Kirchner M: TGFBR1 and TGFBR2 mutations in patients with features of Marfan syndrome and Loeys-Dietz syndrome. Hum Mutat 27: 770-777, 2006.

8. MacCarrick G, Black JH III, Bowdin S, El-Hamamsy I, Frischmeyer-Guerrerio PA, Guerrerio AL, Sponseller PD, Loeys B and Dietz HC III: Loeys-Dietz syndrome: A primer for diagnosis and management. Genet Med 16: 576-587, 2014.
9. Van Hemelrijk C, Renard M and Loeys B: The Loeys-Dietz syndrome: An update for the clinician. Curr Opin Cardiol 25: 546-551, 2010.

10. van de Laar IM, van der Linde D, Oei EH, Bos PK, Bessems JH, Bierma-Zeinstra SM, van Meer BL, Pals G, Oldenburg RA, Bekkers JA, et al: Phenotypic spectrum of the SMAD3-related aneurysms-osteoarthritis syndrome. J Med Genet 49: 47-57, 2012.

11. Loeys BL, Schwarze U, Holm T, Callewaert BL, Thomas GH, Pannu H, De Backer JF, Oswald GL, Symoens $S$, Manouvrier S, et al: Aneurysm syndromes caused by mutations in the TGF-beta receptor. N Engl J Med 355: 788-798, 2006.

12. Van Laer L, Proost D and Loeys BL: Educational paper. Connective tissue disorders with vascular involvement: From gene to therapy. Eur J Pediatr 172: 997-1005, 2013.

13. Malhotra A and Westesson PL: Loeys-Dietz syndrome. Pediatr Radiol 39: 1015, 2009

14. Williams JA, Loeys BL, Nwakanma LU, Dietz HC, Spevak PJ, Patel ND, François K, DeBacker J, Gott VL, Vricella LA and Cameron DE: Early surgical experience with Loeys-Dietz: A new syndrome of aggressive thoracic aortic aneurysm disease. Ann Thorac Surg 83: S757-S763, S785-S790, 2007.

15. Patel ND, Crawford T, Magruder JT, Alejo DE, Hibino N, Black J, Dietz HC, Vricella LA and Cameron DE: Cardiovascular operations for Loeys-Dietz syndrome: Intermediate-term results. J Thorac Cardiovasc Surg 153:406-412, 2017.

16. Krohg-Sørensen K, Lingaas PS, Lundblad R, Seem E, Paus B and Geiran OR: Cardiovascular surgery in Loeys-Dietz syndrome types 1-4. Eur J Cardiothorac Surg 52: 1125-1131, 2017. 